\title{
Hypovirulence-Associated Traits Induced by a Mycovirus of Cryphonectria parasitica Are Mimicked by Targeted Inactivation of a Host Gene
}

\author{
LEI ZHANG, ${ }^{1}$ ALICE C. L. CHURCHILL, ${ }^{2}$ PAM KAZMIERCZAK, ${ }^{1}$ DAE-HYUK KIM, ${ }^{1}$ \\ AND NEAL K. VAN ALFEN ${ }^{1 *}$ \\ Department of Plant Pathology and Microbiology, Texas A\&M University, College Station, Texas $77843,{ }^{1}$ and \\ Molecular Biology Program, Department of Biology, Utah State University, Logan, Utah $84322^{2}$
}

Received 5 May 1993/Revised for modification 8 June 1993/Accepted 9 September 1993

\begin{abstract}
Expression of the Vir2 gene of Cryphonectria parasitica is down-regulated in strains of the fungus containing a double-stranded RNA genetic element that reduces fungal virulence (W. A. Powell and N. K. Van Alfen, Mol. Cell. Biol. 7:3688-3693, 1987). We have sequenced the Vir2 gene and characterized its structure; the mRNA contains a short open reading frame whose product has structural similarities to several fungal pheromones. A null mutant was constructed by homologous recombination to determine the function of the Vir2 gene and whether its disruption resulted in any of the altered phenotypes exhibited by many hypovirulent strains, such as reductions in virulence, pigmentation, and sporulation. The Vir 2 null mutant $(18 \mathrm{dm})$ exhibited a wild-type phenotype with respect to gross colony morphology, growth rate, pigmentation, asexual spore viability, and virulence in apple fruit and chestnut trees. However, numbers of asexual fruiting bodies (pycnidia) and conidia were reduced significantly in comparison with the wild-type strain EP155/2. In sexual crosses of $18 \mathrm{dm}$ with a wild-type strain of the opposite mating type, perithecia (sexual fruiting bodies) developed but were barren. Deletion of the Vir2 gene results in a phenotype that mimics that of many double-stranded-RNA-containing hypovirulent strains; i.e., the null mutant exhibits significant reductions in asexual sporulation and pycnidium production as well as impaired sexual crossing ability. To our knowledge, this is the first report of the partial reproduction of a virus-induced phenotype by deletion of a virus-perturbed host gene.
\end{abstract}

The filamentous fungus Cryphonectria (Endothia) parasitica (Murr.) Barr is responsible for the chestnut blight disease, which destroyed American chestnut forests over a 40-year period during the early part of this century (reviewed in references 3, 4, and 42). Biological control of $C$. parasitica and recovery of infected trees has occurred in Europe by the cytoplasmic transmission of double-stranded RNA (dsRNA) genetic elements between fungal strains. These virus-like elements typically confer a reduction in virulence (hypovirulence) to the fungus and are transmitted from hypovirulent to virulent strains during hyphal anastomosis (fusion of hyphae). In addition to hypovirulence, fungal strains harboring these genetic elements may exhibit an altered colony morphology that includes reduced sporulation and changes in pigmentation $(21$; reviewed in references $4,33,42$, and 52). Reductions in levels of enzymes such as laccase $(9,28$, $40,41)$ and cutinase (53) or of metabolites such as oxalate $(27,28)$ may also occur in hypovirulent strains. The phenomenon of cytoplasmically transmissible hypovirulence in $C$. parasitica is one of the best-documented examples of a naturally occurring form of biological control for plant disease. Our studies are directed towards understanding how dsRNA genetic elements in the fungus cause the attendant changes in host phenotype.

The dsRNA virus-like elements associated with different hypovirulent strains of $C$. parasitica exhibit considerable heterogeneity with respect to genome size, number of dsRNA species, genome concentration, and sequence homology $(20,31,34)$. The largest dsRNA of a European hypovirulent strain and several smaller dsRNA species have

\footnotetext{
*Corresponding author. Electronic mail address: Vanalfen@ tamu.edu.
}

been sequenced $(45,46)$. The largest dsRNA contains two open reading frames (ORFs) which encode polyproteins that proteolytically process themselves $(12,13,46,47)$. A structural resemblance between hypovirulence-associated dsRNAs and replicative forms of positive-strand RNA virus genomes has been noted $(12,22,29,46,51)$. However, the hypovirulence-associated dsRNAs differ from typical plusstrand RNA viruses in that they lack a protein capsid and instead are associated with host-derived cytoplasmic membranous vesicles $(22,26)$.

Recently, a cDNA copy of ORF A of a dsRNA virus was transformed into a wild-type virulent strain of $C$. parasitica and shown to confer partial reductions in pigmentation, conidiation, and laccase accumulation without conferring hypovirulence (10). These results confirm a previous hypothesis $(36,37)$ that the altered phenotypes of dsRNA-containing strains are due to specific effects of the viral dsRNA on the fungus and not to a general debilitation caused by the physical presence of the virus. To firmly establish viral dsRNA as the causal agent of hypovirulence in $C$. parasitica, Choi and Nuss (11) have transformed virulent strains with a full-length cDNA copy of the dsRNA virus. The complete hypovirulence phenotype was conferred, and cytoplasmically replicating dsRNAs were generated from the integrated cDNA copy.

Previous reports have demonstrated that expression of specific poly $(\mathrm{A})^{+}$RNAs and polypeptides of the fungus is altered in dsRNA-containing cells $(8,36,37)$. To understand the mechanism by which dsRNA genetic elements perturb fungal gene expression, knowledge of the host genes that are affected and of their roles in fungal development and virulence is important. Differential plaque filter hybridization was used to identify $C$. parasitica genes whose expression is 
altered in the presence of a virus-encoded dsRNA (36). A genomic DNA fragment coding for one of the genes, Vir2, which is down-regulated in dsRNA-containing strains, was isolated as clone V-2A-1. It should be noted that the Vir designation for this gene was not used with the intent of implying knowledge of function but instead to identify those genes that are transcribed in virulent strains but less so or not at all in dsRNA-containing hypovirulent strains. V-2A-1 hybridizes intensely with total RNA of the wild-type, virulent strain EP155/2 but not with that of the isogenic, hypovirulent, dsRNA-containing strain UEP1 (36).

In this study, the Vir2 gene of $C$. parasitica has been sequenced and its structure has been analyzed. Since Vir2 gene expression is down-regulated in dsRNA-containing strains of the fungus, we were interested in determining if a specific function of the Vir 2 gene could be identified and linked to any aspects of the altered phenotype of hypovirulent strains. The transcribed region of Vir2 was deleted by targeted gene replacement, and the altered phenotype of the resulting null mutant was found to mimic a portion of the virus-induced symptoms.

\section{MATERIALS AND METHODS}

Strains and culture conditions. EP155/2 (a single-spore isolate of EP155; ATCC 38755) and UEP1 are virulent and hypovirulent strains, respectively, of $C$. parasitica. They are isogenic except for the presence of dsRNA in UEP1, which was constructed as previously reported (36). $18 \mathrm{dm}$ is a Vir2 null mutant constructed from EP155/2 as described in this paper. EP44 (mating type a; ATCC 22511) is a wild-type strain with the mating type opposite to that of EP155/2 (mating type A) and was utilized in sexual crosses.

Routine plate cultures were grown on PDAmb (1) at $26^{\circ} \mathrm{C}$ under continuous fluorescent light (250 to $500 \mathrm{~lx})$. Liquid mycelial cultures were grown in EP complete medium (38) as modified by Day et al. (16) but without adjusting the $\mathrm{pH}$. Nucleic acid isolations were done from liquid mycelial cultures. To inoculate liquid cultures, PDAmb plate cultures were grown until the mycelium was almost to the edge of each plate (6 to 8 days), and then each plate culture was homogenized in $100 \mathrm{ml}$ of EP complete medium with a Waring blender and used as the inoculum for 1 liter of the same medium in a Fernbach flask. Cultures were incubated under a constant light intensity $(1,700 \mathrm{~lx})$ on a rotary shaker $(100 \mathrm{rpm})$ at $26^{\circ} \mathrm{C}$ for 2 to 3 days (for DNA isolation) or for the times indicated (for RNA isolation). Cultures were harvested by vacuum filtration through Miracloth (Calbiochem), and the mycelial pad was lyophilized for 24 to $36 \mathrm{~h}$.

Hygromycin-resistant strains were maintained on media containing $50 \mu \mathrm{g}$ of hygromycin $\mathrm{B}$ per $\mathrm{ml}$, and benomylresistant strains were maintained on media containing $0.5 \mu \mathrm{g}$ of benomyl per ml. Tests for prototrophic growth were performed on minimal medium (38). Cultures analyzed for asexual sporulation were grown on either PDAmb or $8 \%$ malt extract agar (MEA) (80 g of Difco malt extract and $20 \mathrm{~g}$ of Difco agar per liter). Production of pycnidia (asexual fruiting bodies) was assessed on either 1.5 or $8 \%$ MEA $(35 \mathrm{ml}$ of agar per plate). Growth of $C$. parasitica on MEA reduces formation of aerial hyphae, thereby allowing quantitation of pycnidia.

Stock cultures were maintained either on PDAmb slants at $4^{\circ} \mathrm{C}$ or on silica gel as previously described (14). Escherichia coli XL1-Blue (Stratagene) was the recipient for bacterial transformation and routine propagation of plasmids.

Fungal nucleic acid isolation. Genomic DNA was isolated

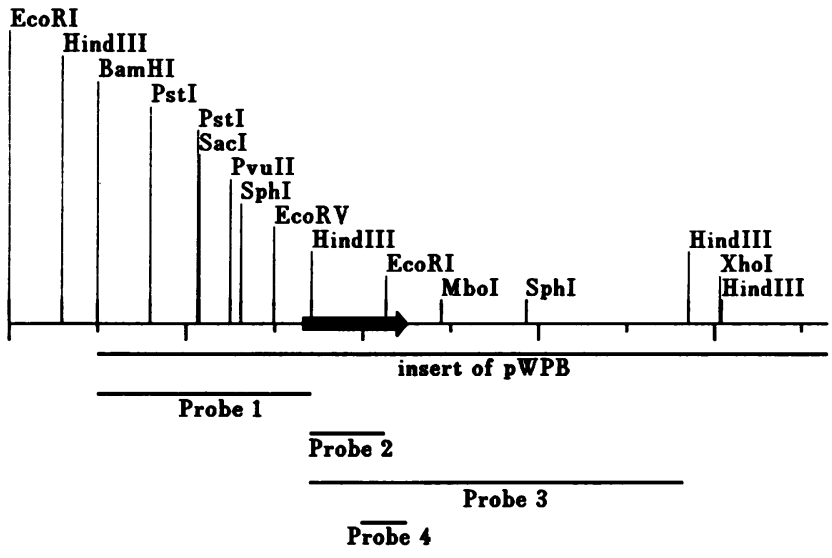

$1^{1000}, 1_{1}^{2000}, 1_{1}^{3000}, 1_{1}^{4000 \quad(b p)}$

FIG. 1. Partial restriction map of the Vir2 transcribed region and adjacent sequences. The location and direction of Vir2 transcription are indicated by the arrow. The four fragments labeled probe 1 , probe 2 , probe 3 , and probe 4 were used for hybridization in Northern and Southern blot analyses. Probe 1 is a 1.2-kb BamHIHindIII fragment which contains $47 \mathrm{bp}$ of the $5^{\prime}$ region of the Vir2 mRNA and upstream nontranscribed regions. Probe 2 is a 426-bp HindIII-EcoRI fragment containing a major portion of the transcribed region of Vir2. Probe 3 is a 2.1-kb HindIII fragment which includes the probe 2 and probe 4 regions as well as downstream nontranscribed regions. Probe 4 consists of 241 bp of the $3^{\prime}$ end of the Vir2 transcribed region (nt 472 to 713 in Fig. 3). The genomic DNA insert in pWPB is also indicated.

by a modification of the protocol of Yelton et al. (55) as previously described (57).

Total RNA was isolated as previously described (36). Poly(A) ${ }^{+}$RNA was isolated by using a Pharmacia mRNA purification kit according to the instructions of the manufacturer.

Southern and Northern (RNA) blot analyses. Southern and Northern blot analyses were performed as previously described (36). GeneScreen Plus (DuPont) was used as the hybridization membrane, and the formamide procedure suggested by the manufacturer was followed. $\left[{ }^{32} \mathrm{P}\right] \mathrm{dCTP}$-labeled probes were prepared by using a random-primed DNAlabeling kit (Boehringer Mannheim Biochemicals).

Characterization of $V i r 2$ gene expression. A 4.2-kb genomic DNA fragment was subcloned from V-2A-1 (36) into pBluescript $\mathrm{KS}(+)$ (Stratagene) and designated pWPB. The location of the Vir2 transcriptional region within this fragment was determined by digesting the $4.2-\mathrm{kb}$ fragment into seven fragments ranging in size from 0.4 to $0.8 \mathrm{~kb}$, using a variety of restriction enzymes. Each fragment was isolated from gel slices by using GENECLEAN (Bio 101), ${ }^{32} \mathrm{P}$ labeled by random priming, and hybridized to Northern blots of total RNA to determine which fragment(s) had homology to Vir2 transcripts.

A time course of Vir2 gene expression was conducted by isolating total RNA at 24-h intervals (beginning at day 2) from EP155/2 cultures grown in liquid medium for 8 days. Total RNA $(7 \mu \mathrm{g})$ from each time point was separated by electrophoresis on a $1.5 \%$ agarose gel with glyoxal-dimethyl sulfoxide as the denaturing agent (5), Northern blotted, and hybridized with ${ }^{32} \mathrm{P}$-labeled probe 4 (Fig. 1). Hybridization signals on the Northern blot were quantified with the aid of 
an LKB densitometer (Ultroscan XL enhanced laser densitometer).

DNA sequencing and analysis. Genomic DNA was sequenced by using the M13 cloning/dideoxy sequencing kit (Bethesda Research Laboratories). Vir2 cDNA clones were sequenced by using the Sequenase DNA-sequencing kit (version 2.0; U.S. Biochemical). Double-stranded plasmid template DNA was prepared by the methods of Del Sal et al. (18). The sequence reported was determined by sequencing both strands.

S1 nuclease mapping and primer extension studies. The location of the $5^{\prime}$ end of Vir 2 was determined by $\mathrm{S} 1$ nuclease analysis, which was performed according to the methods of Ausubel et al. (5). A synthesized oligonucleotide complementary to nucleotides (nt) 153 to 173 of the Vir2 sequence (see Fig. 3) was $5^{\prime}$ end labeled with ${ }^{32} \mathrm{P}$ and annealed to a single-stranded M13mp19 vector containing the 215-bp EcoRV-HindIII fragment (Fig. 1). The oligonucleotide primer was extended with Klenow fragment, and the doublestranded molecule was digested with EcoRV. The singlestranded DNA probe was isolated, after separation on a $1.2 \%$ alkaline agarose gel, and used for $S 1$ analysis. In the $S 1$ analysis, $50 \mu \mathrm{g}$ of total RNA or $0.5 \mu \mathrm{g}$ of poly(A) ${ }^{+}$RNA, from 7-day-old liquid cultures, was used.

The location of the $3^{\prime}$ end of Vir2 was determined by S1 nuclease analysis (5). A uniformly ${ }^{32} \mathrm{P}$-labeled antisense RNA was synthesized by using T7 RNA polymerase. A 313-bp EcoRI-MboI fragment (Fig. 1) was cloned into pBluescript $\mathrm{KS}(+)$ at the EcoRI-BamHI site. The fragment containing the insert was excised with PvuII and EcoRI, eluted from a $1.2 \%$ agarose gel, phenol-chloroform extracted, ethanol precipitated, and used as template in the RNA transcription reaction. The transcription reaction was performed according to the pBluescript II Exo/Mung DNA sequencing system instruction manual (Stratagene). The radiolabeled probe was further purified by electrophoresis in a $6 \%$ denaturing acrylamide gel.

Primer extension studies were performed according to the methods of Ausubel et al. (5). The same oligonucleotide that was used for the $S 1$ nuclease analysis of the $5^{\prime}$ end of Vir2 was used for the primer extension experiment. RNA was isolated from 7-day-old liquid cultures.

Vir2 cDNA clone isolation. Single-stranded cDNA of EP155/2 was prepared as previously described (57).

The polymerase chain reaction (PCR) was used to isolate cDNA clones of the Vir2 gene. Aliquots of 1 to $4 \mu$ l of single-stranded DNA were used in each PCR. The PCRs were performed by using the Repliprime DNA amplification system (DuPont) according to the recommendations of the manufacturer. Denaturation was at $94^{\circ} \mathrm{C}$ for $30 \mathrm{~s}$, and annealing was at $55^{\circ} \mathrm{C}$ for $2 \mathrm{~min}$ with ramping to $72^{\circ} \mathrm{C}$ in $30 \mathrm{~s}$ and continuing at $72^{\circ} \mathrm{C}$ for $2 \mathrm{~min}$ for a total of 35 cycles. The final cycle of incubation at $72^{\circ} \mathrm{C}$ was for $10 \mathrm{~min}$. The $3^{\prime}$ primer used was the same as that used to make cDNA. Two $5^{\prime}$ primers were synthesized. Primers 1 and 2 are nt 146 to 171 and 461 to 487 , respectively (see Fig. 3). The PCR products of interest were excised from a $1.5 \%$ agarose gel and cloned into pBluescript $\mathrm{KS}(+)$.

Construction of plasmids for gene replacement and complementation. The gene replacement plasmid pDM18 was constructed as illustrated in Fig. 5. An 880-bp PvuII-EcoRI fragment from pWPB was removed and replaced with the hygromycin phosphotransferase gene (hph) of $E$. coli (24) as a selectable marker. The $h p h$ gene, with trpC promoter and terminator sequences of Aspergillus nidulans, was retrieved from pDH25 (15) (provided by Genentech, Inc., San Fran- cisco, Calif.) by restriction with the enzymes $S s p \mathrm{I}$ and $N r u \mathrm{I}$. The $h p h$ sequence was ligated to the Vir2 gene flanking regions from pWPB and pBluescript $\mathrm{KS}(+)$ to produce the gene replacement plasmid pDM18. pDM18 was restricted with PvuII, and the resulting 7.6-kb fragment, denoted fDM18, was eluted from an agarose gel with GENECLEAN. This linear fragment was used for fungal transformation of strain EP155/2. To construct a plasmid for complementation of Vir2 in strain dm18, from which Vir2 was deleted, a 2.6-kb SalI fragment from pSV50 (54) containing the benomyl resistance gene was ligated into the $X$ hoI site of pWPB. This plasmid (pCV2) was linearized with $\mathrm{XbaI}$ and transformed spheroplasts of dm18.

Fungal transformation. Spheroplast preparation, transformation, and selection for drug-resistant transformants were conducted under optimized conditions as previously described (14). Spheroplasts were transformed with $10 \mu \mathrm{g}$ of linear gene or plasmid.

Phenotype of null mutant. In all assays used to characterize the phenotype of the Vir2 null mutant, the wild-type strain EP155/2 and one to three pDH25 control transformants were included for comparison. Mitotic stability was initially examined by serial transfers of mycelial inoculum as previously described (14). Further examination of mitotic stability was by germination of conidia, isolated from cultures grown under nonselective conditions, on PDAmb with or without hygromycin B. The radial growth rate of colonies was measured after growth for 5 to 7 days on minimal medium, $1.5 \%$ MEA, or PDAmb with or without hygromycin B. Tests for virulence on apple fruit were performed as previously described (21). Virulence tests on chestnut trees were performed according to the procedures of Rigling et al. (40). The trees had a diameter at breast height of approximately $2.5 \mathrm{~cm}$. They were grown and maintained after inoculation within a growth chamber at $30^{\circ} \mathrm{C}$ with a $16-\mathrm{h}$ light/8-h dark photoperiod. The lesion area was measured at $7,12,19,26$, and 34 days after inoculation.

Asexual sporulation was assessed after growth of cultures on PDAmb for 2 or 3 weeks. Spores were harvested by flooding each petri dish culture (100 by $15 \mathrm{~mm}$ ) with sterile distilled $\mathrm{H}_{2} \mathrm{O}$ and dislodging conidia with a glass rod; each plate was rinsed once with an additional aliquot of $\mathrm{H}_{2} \mathrm{O}$. The spore solution was filtered through $20-\mu \mathrm{m}$ nylon mesh (Spectrum Medical Industries, Inc.) to remove mycelial debris, and the spore concentration was determined by counting with a hemacytometer.

Pycnidium production was assessed on either 1.5 or $8 \%$ MEA. Isolates were compared by counting the pycnidia within a central circular 7-cm-diameter region of the petri dish (38.5- $\mathrm{cm}^{2}$ area) with the aid of a dissecting microscope after growth for 2,3 , or 4 weeks. To estimate the average number of spores per pycnidium, the $38.5-\mathrm{cm}^{2}$ area of medium was cut from the petri dish and spores were harvested as described above. For all experiments, a minimum of three replicates were included for each treatment, and the data were analyzed by analysis of variance by the SAS GLM procedure (44) with Duncan's multiple range test.

Sexual crosses were performed on sterile chestnut wood according to the methods of Anagnostakis (2). The Vir2 null mutant $18 \mathrm{dm}$ was crossed with the wild-type strain EP44 of opposite mating type. Control crosses included EP155/2 with EP44 and EP44 with 102pDH25 (a control transformant).

Nucleotide sequence accession number. The sequence data reported for the Vir2 gene have been assigned GenBank accession number L11143. 


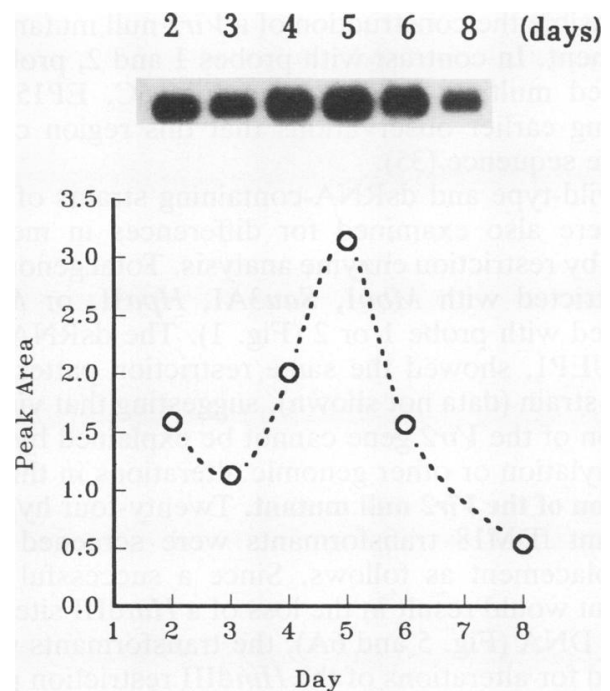

FIG. 2. Time course of Vir2 gene expression by EP155/2 grown in liquid culture. Total RNA was extracted from mycelia at the indicated times. (Top) Autoradiogram of the Northern blot. Each lane contains $7 \mu \mathrm{g}$ of total RNA. The blot was hybridized with ${ }^{32} \mathrm{P}$-labeled probe 4 (Fig. 1), which represents 241 bp of the $3^{\prime}$ transcriptional region of Vir2. Equal loading of RNA was confirmed in a parallel blot hybridized with an rDNA probe from $N$. crassa (data not shown). (Bottom) Densitometric scan of the autoradiogram in the top panel.

\section{RESULTS}

Location and direction of transcription of Vir2. A partial restriction map of the 4.7-kb genomic DNA fragment containing the Vir2 transcribed region is shown in Fig. 1. By Northern blot analysis, the coding region of Vir2 was localized to the 426-bp HindIII-EcoRI fragment and the adjacent $5^{\prime}$ and $3^{\prime}$ regions (data not shown). The direction of transcription was determined to be from the HindIII to the
EcoRI site (data not shown). These results were confirmed by the cDNA sequence data discussed below.

Time course of expression of Vir 2 mRNA. The time course of Vir2 mRNA expression in the wild-type, virulent EP155/2 strain is shown in Fig. 2. Vir2 mRNA was detected by Northern blot analysis at all points examined. mRNA levels were greatest at 5 days after inoculation, by which time the stationary growth phase had been reached. Vir2 mRNA was not detected by Northern analysis in the dsRNA-containing strain UEP1 during the same time course (data not shown).

Vir 2 gene sequence and structure analysis. The genomic DNA sequence of the Vir 2 transcribed and adjacent regions is shown in Fig. 3. Several sequences which may play important roles in gene expression and regulation were identified. A potential CCAAT sequence (CAAAT) is $97 \mathrm{bp}$ upstream from the first transcription start point at position 123. A Goldberg-Hogness box, TATAAATA, at positions 75 to 82 is located 40 bp upstream from the first transcription start site. A putative initiator element (49), TATTCT, is located $13 \mathrm{bp}$ upstream from the first transcription start site. A putative capping sequence, TCATCAG, is found at nt 121 around the first sites of transcription initiation. The region around the first ATG codon is TCAAAATG, a recognized sequence for protein translation start sites in some filamentous fungi $(6,25)$. Four small in-frame ORFs (corresponding to $23,31,13$, and 11 amino acids, respectively), each starting with an ATG codon and followed by a TAA stop codon, are located at positions $197,284,479$, and 539 , respectively.

S1 nuclease analysis (Fig. 4) indicated the presence of multiple transcription start sites at positions 123 to 132 . The predominant sites are A's located at positions 123, 126, and 129 , which are 74,71 , and 68 bases, respectively, upstream of the first ATG codon (Fig. 3). A primer extension experiment, in which the same primer used for S1 nuclease analysis was utilized, confirmed these results (data not shown). Since the same results were obtained by primer extension and S1 nuclease analysis, it is unlikely that the multiple start sites are artifacts. In both experiments, total RNAs of the dsRNA-containing UEP1 strain and the Vir2

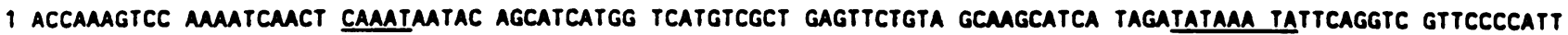

101 TGGTATTCTG TCTGTCCTCC TCATCAGCAT CACAMACCTC ACAAACACTC TACAGMaCA TCTTCTTAAG CTTCACGCTT CAMACCAMCC ATCAMATGC

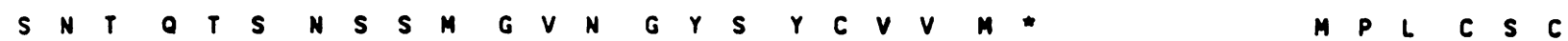

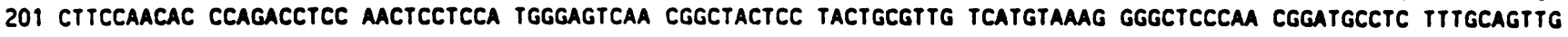

K R $R$ K $S$ S S $T$ T I

301 CAAGCGGAAG AGTTCTACAM TACACCACAT GGTTGGTAGT CCGACGATAC CAGCCATCGC TAGCCACTTC GCCACGTAaC MaCACGCAaG agCCTACCGA

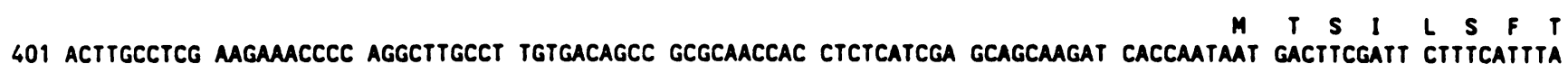
C R L S H * M R T D C C G G F A M L *

501 CCTGCCGCCT TTCTCATTAA CCCTGCGGTG ACGACCGGAT GCGAACGGAC TGCGGCGGAT TTGCGATGTT GTAATCTATT GGCCATGTTT TCGGAATTCA

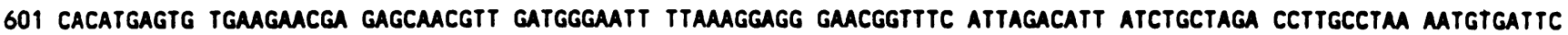

\section{AGTCATCCTC CAG}

FIG. 3. Nucleotide sequence of the Vir 2 transcriptional region and the adjacent 5 ' nontranscribed regions. The first transcription start site is at position 123, and transcription ends at residue 713 (polyadenylation site). Underlined sequences are as follows: CCAAT motif (CAAAT, bases 21 to 25), TATA box motif (TATAAATA, bases 75 to 82), putative initiator sequence (TATTCT, bases 104 to 109), putative capping site (TCATCAG, bases 121 to 127) around the transcription start sites, and translational consensus sequence (TCAAAATG, bases 192 to 199) around the first ATG site. The protein sequences encoded by the four ORFs were deduced from the DNA sequence. A CAAX motif (CVVM), representing a possible site of prenylation, is located at the carboxyl-terminal end of ORF 1 . Asterisks indicate the locations of stop codons. No introns were detected within the transcribed sequence. 


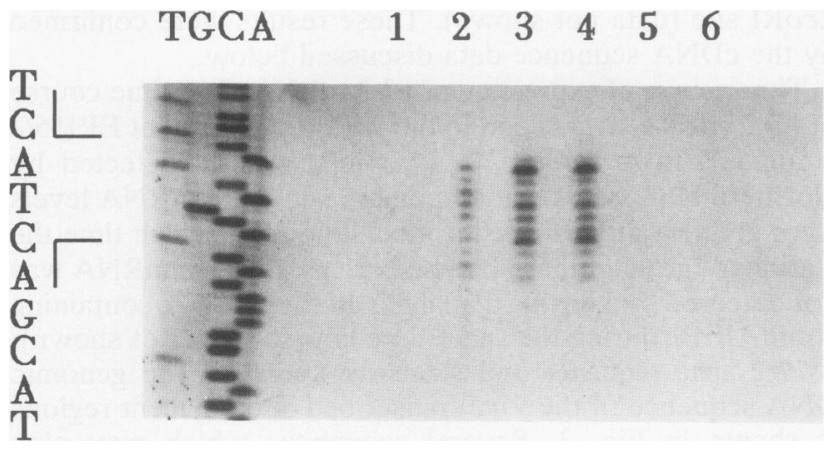

FIG. 4. S1 nuclease analysis of the $5^{\prime}$ end of Vir2. A ${ }^{32} \mathrm{P}-5^{\prime}$-endlabeled 215-base single-stranded DNA complementary to the EcoRV-HindIII fragment (Fig. 1) was synthesized and used as a probe in S1 nuclease analysis. Lanes: 1, no RNA; 2, EP155/2 poly $(\mathrm{A})^{+}$RNA $(0.5 \mu \mathrm{g}) ; 3$ and 4 , two separate preparations of EP155/2 total RNA $(50 \mu \mathrm{g}) ; 5,18 \mathrm{dm}$ total RNA $(50 \mu \mathrm{g}) ; 6$, UEP1 total RNA (50 $\mu \mathrm{g}) ; \mathrm{T}, \mathrm{G}, \mathrm{C}$, and A, DNA sequencing ladders. The same M13 DNA and primer were used in the sequencing reaction as in the single-stranded probe synthesis.

null mutant $18 \mathrm{dm}$ were used as negative controls to rule out any nonspecific signals.

To determine the location of the $3^{\prime}$ end of the Vir2 transcript, a ${ }^{32}$ P-labeled antisense RNA complementary to the 313-bp EcoRI-MboI fragment (Fig. 1) was used to perform an S1 nuclease protection assay. A 120-base fragment was detected (data not shown), suggesting that the $3^{\prime}$ end of Vir2 is 120 bases downstream from the EcoRI site. The poly $(\mathrm{A})^{+}$addition site was defined as nucleotide $\mathrm{G}$ at position 713 (Fig. 3) by sequencing seven independent cDNA clones. cDNAs were created and amplified by PCR using an oligo(dT)-containing $3^{\prime}$ primer paired either with $5^{\prime}$ primer 1 (nt 146 to 171 ) or $5^{\prime}$ primer 2 (nt 461 to 487 ). A single 600-bp product was generated from amplifications by using the $5^{\prime}$ primer $1-3^{\prime}$ primer pair. The PCR product obtained from the $5^{\prime}$ primer 2-3' primer pair showed a major band of $250 \mathrm{bp}$ in length. Two independent clones from the primer 1 cDNA and five clones from the primer 2 cDNA were sequenced. All seven sequences showed the same poly(A) ${ }^{+}$ addition site. No introns were found by comparing the cDNA sequence with the genomic DNA sequence. Although the cDNA clones were not full length, the fact that the primer 1 sequence (nt 146 to 171) overlapped with the primer used in the $5^{\prime}$ end $S 1$ nuclease analysis (nt 153 to 173) confirmed that there were no introns within the region from the transcription initiation site to the location of primer 1 . These data are in agreement with the size of the Vir2 transcript, which was determined to be approximately 650 bases by Northern analysis.

Copy number of the Vir2 gene. Total genomic DNA of EP155/2 or UEP1 was restricted with BamHI, EcoRI, HindIII, or both EcoRI and HindIII. Southern blots were hybridized with either probe 1 (Fig. 1), which contains 47 bp of the $5^{\prime}$ transcribed sequence of Vir2 as well as upstream regions, or probe 2 (Fig. 1), which contains the sequence representing the major transcribed region of Vir2. For each probe, identical banding patterns were observed for both strains (data not shown). A single band was identified for each digest at the position predicted by sequence analyses and genomic DNA clone restriction studies. These results suggested that the Vir2 gene was present as a single copy in the genomes of both EP155/2 and UEP1 and, consequently, made feasible the construction of a Vir2 null mutant by gene replacement. In contrast with probes 1 and 2, probe 3 (Fig. 1) showed multiple bands (as in Fig. 6C, EP155/2 blot), supporting earlier observations that this region contains a repetitive sequence (35).

The wild-type and dsRNA-containing strains of $C$. parasitica were also examined for differences in methylation patterns by restriction enzyme analysis. Total genomic DNA was restricted with MboI, Sau3AI, HpaII, or MspI and hybridized with probe 1 or 2 (Fig. 1). The dsRNA-infected strain, UEP1, showed the same restriction patterns as the EP155/2 strain (data not shown), suggesting that viral downregulation of the Vir2 gene cannot be explained by differential methylation or other genomic alterations in this region.

Isolation of the Vir2 null mutant. Twenty-four hygromycin B-resistant fDM18 transformants were screened for Vir2 gene replacement as follows. Since a successful gene replacement would result in the loss of a HindIII site from the genomic DNA (Fig. 5 and 6A), the transformants were first examined for alterations of the HindIII restriction pattern in Southern blots of total genomic DNA (data not shown). One transformant, $18 \mathrm{dm}$, was identified as a putative Vir2 null mutant and further studied by detailed Southern blot analysis. Figure 6B shows the Southern blots of $18 \mathrm{dm}$ genomic DNA when hybridized with either probe 1 or the $h p h$ gene probe (Fig. 6A). The restriction patterns on the Southern blots fit the predicted band sizes, although the EcoRI digestion apparently was not complete.

Since the $0.42-\mathrm{kb}$ region corresponding to probe 2 (Fig. 1) was deleted from the genomic DNA of $18 \mathrm{dm}$, no Vir2 bands were detected in Southern blots of 18dm DNA hybridized to this probe (Fig. 6C). The faint bands visible on the EP155/2 and $18 \mathrm{dm}$ blots hybridized with probe 2 represent homology of the probe to a second distinct gene, Virl (56). To prove that the absence of Vir2 bands in the 18dm-probe 2 Southern blot was not due to an experimental artifact, probe 3 , containing a repeat sequence distributed among several chromosomes (35), was hybridized with an identical blot (Fig. 6C). Probe 3 detected several bands in $18 \mathrm{dm}$ total DNA that represent repetitive DNA sequences also found in EP155/2. Other bands detected by probe 3 on the $18 \mathrm{dm}$ blot changed mobility relative to the EP155/2 bands because of the replacement of the 880-bp PvuII-EcoRI fragment with the $h p h$ gene. The restriction mapping studies indicated that the 880-bp fragment of the Vir2 gene was deleted in strain $18 \mathrm{dm}$ by homologous recombination and replaced with one copy of the $h p h$ gene with a $\operatorname{trp} C$ promoter and terminator.

Northern blot analysis showed that Vir2 mRNA was not expressed in 18dm. However, two independent pDH25 transformants which showed stable hygromycin $B$ resistance exhibited Vir2 mRNA expression comparable to that of the wild-type strain EP155/2 (data not shown).

Phenotype of the Vir2 null mutant. 18dm and the pDH25 control transformants were mitotically stable and maintained resistance to hygromycin $B$ in the absence of the drug for up to at least 3 weeks of active growth. Growth rate and pigmentation of the Vir2 null mutant on minimal and complete media, as well as colony morphology, were comparable to those of EP155/2 and the pDH25 control transformants. Viability of $18 \mathrm{dm}$ conidia was not significantly different from that of EP155/2. In both the apple fruit and chestnut tree virulence tests, lesion size caused by the Vir2 null mutant was comparable to or greater than that caused by EP155/2 and always significantly greater than that caused by UEP1, indicating that deletion of the Vir2 transcribed region had no measurable effect on virulence (data not shown). Inocula of 

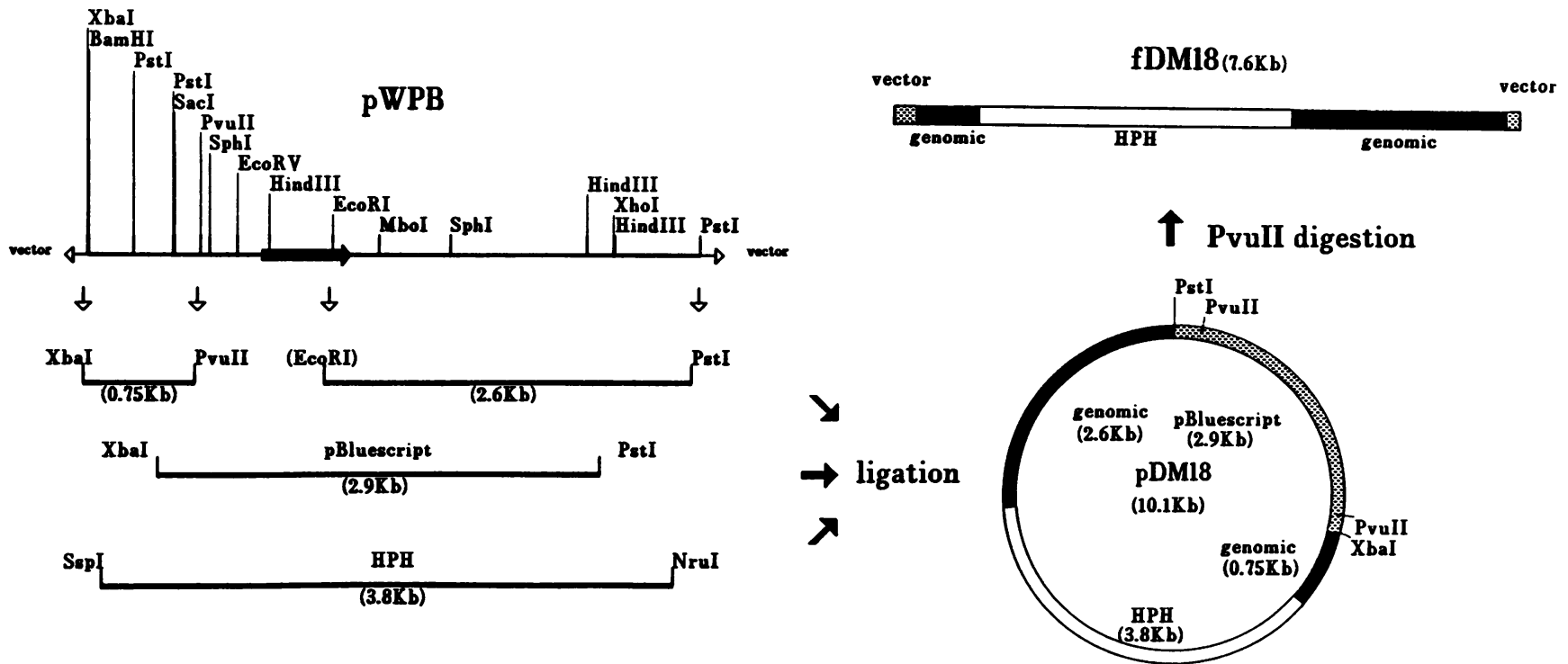

FIG. 5. Construction of pDM18 for the Vir2 gene replacement. The XbaI-PvuII (0.75-kb) and the EcoRI-PstI (2.6-kb) genomic DNA fragments from pWPB were used as the $5^{\prime}$ and $3^{\prime}$ flanking regions, respectively, in this construction. After restriction enzyme digestion, the fragments were purified by gel electrophoresis and retrieved by GENECLEAN. The EcoRI site was blunted by the fill-in activity of Klenow. The $E$. coli hph gene, with an $A$. nidulans trpC promoter and terminator, was retrieved from pDH25 by restriction with $S s p I$ and NruI. The two genomic DNA fragments and the $h p h$ gene were ligated to pBluescript $\mathrm{KS}(+)$ that had been restricted with $X b a \mathrm{I}$ and PstI, to construct pDM18. pDM18 was digested with PvuII, and the 7.6-kb linear DNA fragment fDM18, containing the $h p h$ gene and Vir2 gene flanking regions, was used for transformation of $C$. parasitica.

$18 \mathrm{dm}$ and a control transformant reisolated from chestnut tree cankers at the completion of the experiment showed stable resistance to hygromycin $B$.

Quantitative analysis of sporulation and pycnidium production by $18 \mathrm{dm}$ revealed statistically significant reductions in both functions when compared with EP155/2 and the three pDH25 transformants. In the first set of four independent experiments conducted, sporulation was assessed by growth of each fungal isolate on PDAmb. Under these conditions, sporulation was reduced in the Vir2 null mutant to $8.7,0.2$, 0.1 , and $0.4 \%$ of the wild-type level represented by EP155/2 and the $\mathrm{pDH} 25$ transformants. Figure 7A shows data from experiment 3 that are representative of the effect of deletion of the Vir2 gene on sporulation.

Pycnidium production was assessed in four independent experiments by the growth of fungal isolates on $1.5 \%$ MEA. Under these conditions, pycnidium formation was reduced in $18 \mathrm{dm}$ to $69.5,22.1,46.0$, and $32.1 \%$ of that in control isolates. Figure 7B shows data from experiment 2 that are representative of the effect of deletion of the Vir2 gene on pycnidium production. For determination of both spore and pycnidium numbers, similar results were obtained when single-spore isolates were tested in comparison with the primary transformant. The morphology of the pycnidia produced by $18 \mathrm{dm}$ appeared normal by gross comparison, with the aid of a dissecting microscope, with wild-type and pDH25 transformant control strains.

In an attempt to determine if reduced pycnidium formation was the sole factor causing reduced sporulation in $18 \mathrm{dm}$, two additional experiments were conducted. The average number of spores per pycnidium was calculated by assessing both sporulation and pycnidium production on a single medium, $8 \%$ MEA, to determine if pycnidia from $18 \mathrm{dm}$ produced fewer spores on average than wild-type and control strains. In one experiment, pycnidium production and the average number of spores per pycnidium were both reduced in the Vir2 null mutant by 3.1- and 3.3-fold, respectively, almost completely accounting for the 11.3-fold reduction in sporulation of $18 \mathrm{dm}$ compared with controls. However, when the experiment was repeated, the $71.2 \%$ reduction in sporulation by $18 \mathrm{dm}$ corresponded to an approximately equivalent reduction in pycnidium production. No effect on the average number of spores per pycnidium was detected in the second experiment. Therefore, it is unclear whether deletion of the Vir2 gene causes a reduction in sporulation solely by reducing the numbers of pycnidia formed or whether other processes that affect spore number are perturbed as well.

Sexual crosses were performed to confirm the linkage between reduced sporulation and pycnidium number in $18 \mathrm{dm}$ with Vir2 gene inactivation. The perithecia that formed from the crosses of $18 \mathrm{dm}$ with the wild-type strain EP44, however, were barren; i.e., perithecia were present, but few to no ascospores were produced. In contrast, control crosses utilizing two wild-type strains (EP44 with EP155/2) or a control transformant and a wild-type strain (102pDH25 with EP44) resulted in the production of normal perithecia and ascospores.

Complementation of Vir2 by transformation of this gene into strain $18 \mathrm{dm}$ was done to determine if the wild-type level of asexual sporulation could be restored. A total of 16 random benomyl-resistant transformants of $18 \mathrm{dm}$ by $\mathrm{pCV} 2$ were selected and separately analyzed for asexual sporulation levels, presence of Vir 2 mRNA by Northern blots, and integration of pCV2 into the genome by Southern analysis. Each of the transformants showed a different fragment pattern when their DNA was hybridized with the 426-bp probe 2 described in Fig. 1, indicating random integrations of pCV2 into each transformant. Expression of Vir2 could be detected by Northern blots in 14 of the 16 transformants. Asexual sporulation was restored to wild-type levels in 5 of these 14 transformants. The two transformants which did not 
A.

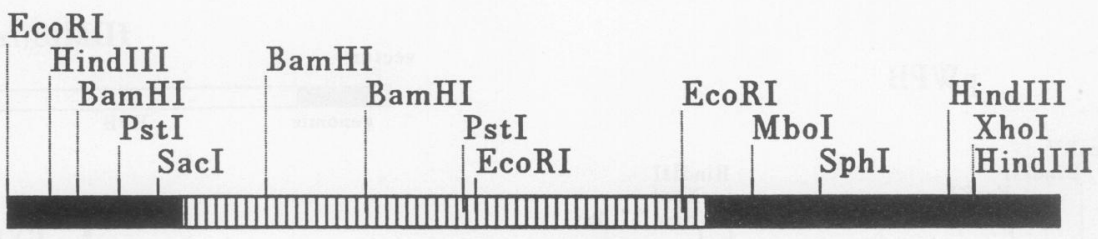

parent DNA

HPH gene

parent DNA

Probe 1

Probe 3

Probe HPH

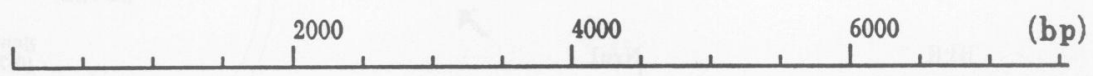

B.

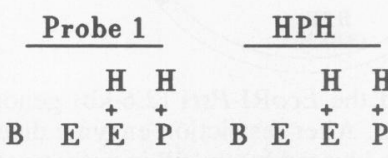

C.

$\mathrm{H}$
+
$\mathbf{P}$

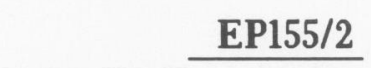

$18 d m$
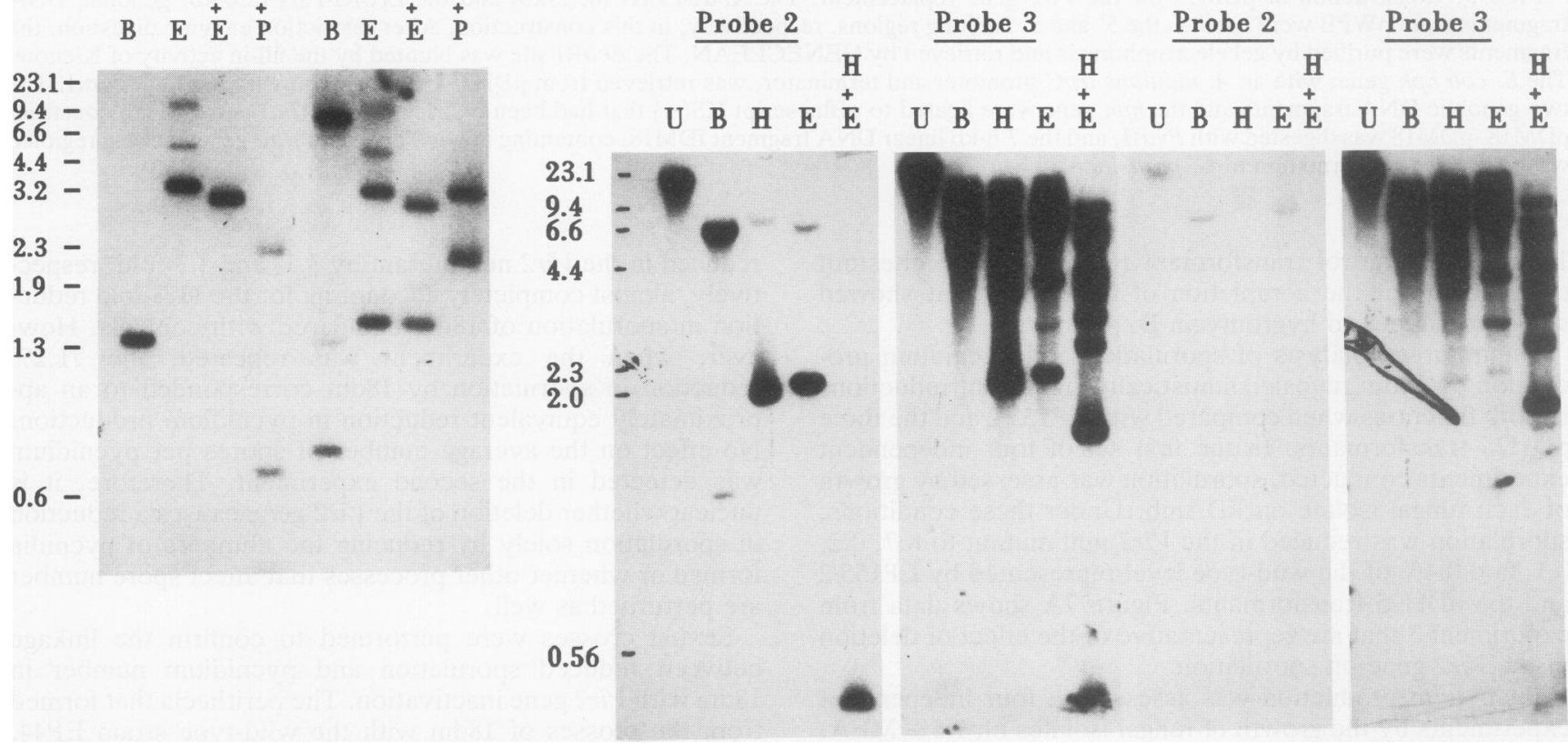

FIG. 6. Southern blot analyses of $18 \mathrm{dm}$ genomic DNA. (A) Partial restriction map of the gene replacement site of $18 \mathrm{dm}$. Probes 1,2 , and 3 used in this set of experiments are the same as those shown in Fig. 1. However, here probes 1 and 3 are shown truncated to indicate the existing regions of homology with the parental DNA after deletion and replacement of the Vir2 transcriptional region with the $h p h$ gene. (B) Southern blot analysis of the null mutant $18 \mathrm{dm}$ when hybridized with either probe 1 or the $h p h$ probe. (C) Southern blot analyses of EP155/2 and the null mutant $18 \mathrm{dm}$ hybridized with either probe 2 (Fig. 1) or probe 3 , which contains a repetitive sequence. Abbreviations: $U$, uncut DNA; B, BamHI; H, HindIII; E, EcoRI; P, PstI.

express Vir2 sporulated at the reduced levels typical of $18 \mathrm{dm}$.

\section{DISCUSSION}

We report here the sequence, structure, and possible biological function of a fungal gene of $C$. parasitica, Vir2, expression of which is down-regulated in the presence of a virus-encoded dsRNA (36). No detectable differences were found in the hybridization patterns of EP155/2 and UEP1 DNAs restricted with methylation-sensitive and -insensitive restriction enzymes. Therefore, we have no evidence for differential methylation or other genomic alterations at sites within or near the Vir2 gene of UEP1 as an explanation for how viral dsRNA might down-regulate this gene.

Vir2 gene structure. Sequences resembling CCAAT and TATA boxes are present in the 5 ' nontranscribed region of Vir2 (TATAAATA). These sequences show similarities with the promoter region of another highly expressed $C$. parasitica gene, that for cryparin (TATAAA) (57). The $C$. parasitica laccase gene also contains a similar TATA box sequence, TATATAAA (9).

The regions around the transcription initiation sites of both the Vir2 and cryparin genes are also very similar 

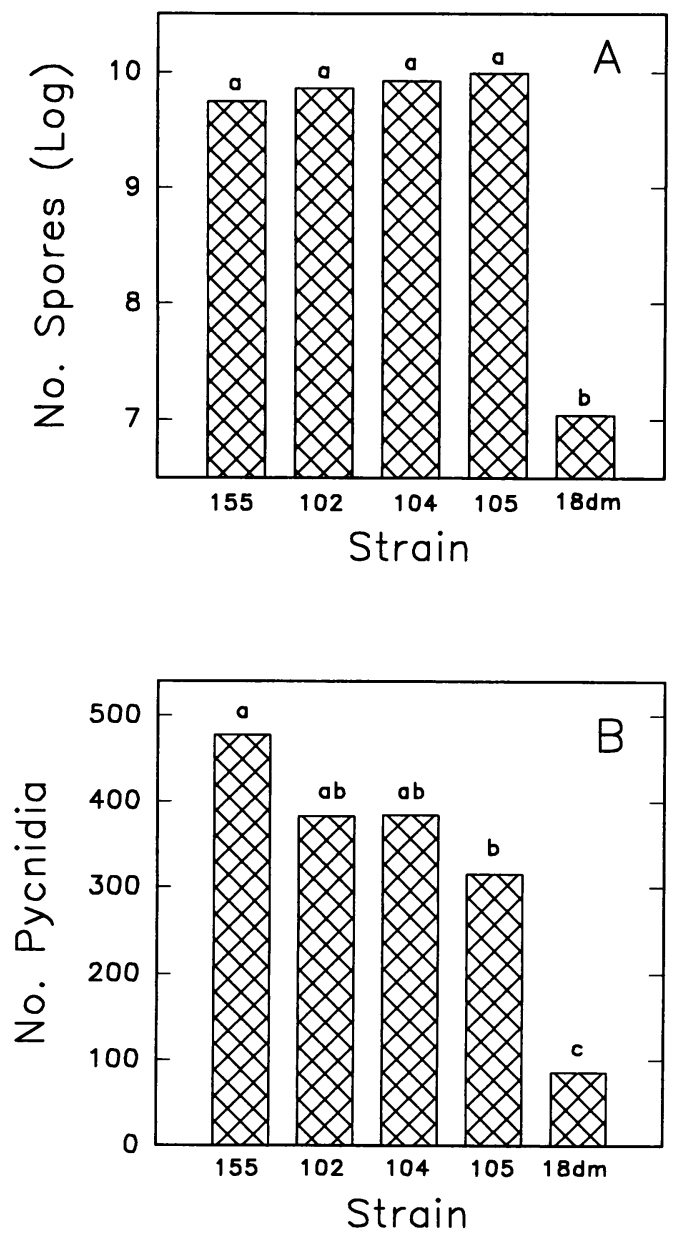

FIG. 7. Effect of deletion of the Vir2 gene on sporulation and pycnidium production by $C$. parasitica. (A) Log of the average number of spores produced per petri dish $(100$ by $15 \mathrm{~mm})$ for each isolate tested. Each bar represents the average of three replicates. Similar results were observed in five additional experiments, each showing a statistically significant reduction in spore number by the Vir2 null mutant $18 \mathrm{dm}$ in comparison with the wild-type and $\mathrm{pDH} 25$ controls $(P<0.002)$. (B) Average number of pycnidia produced within a $38.5-\mathrm{cm}^{2}$ area for each isolate tested. Each bar represents the average of three replicates. This experiment is representative of the results observed in five additional experiments, each showing a significant reduction in pycnidium number by the Vir2 null mutant $18 \mathrm{dm}$ in comparison with the wild-type and pDH25 controls $(P<$ 0.03). In most experiments, the number of pycnidia produced by strain $105 \mathrm{pDH} 25$ was not significantly different from that produced by strain EP155/2. Data were analyzed by analysis of variance with Duncan's multiple range test. For each histogram, bars labeled with the same letters are not significantly different from one another $(P$ values are listed above). Isolate abbreviations: 155, EP155/2 (wildtype control); 102, 102pDH25; 104, 104pDH25; 105, 105pDH25 (pDH25 control transformants); $18 \mathrm{dm}$, Vir2 null mutant.

(TCCTCCTCATCAG for Vir2 and TCCTCCTTCTTCATCA for the cryparin gene). For the Vir2 gene, $70 \%$ of the sequence between the TATA box and the first transcription start site is pyrimidine rich (28 of 40 residues). In many instances, such pyrimidine-rich regions are a common feature of sites of transcription initiation in filamentous fungi $(6$, 25). The sequence around the first transcription initiation site of Vir2, TCATCAG, is equivalent to the putative Neurospora crassa capping consensus sequence PyCATCAPu (43).
With only a few exceptions, translation of most eukaryotic genes begins at the first ATG site after transcription initiation (30). In filamentous fungi, as in higher eukaryotes, the sequence immediately upstream of the ATG codon is instrumental for correct translation initiation (25). The consensus sequence for this region is TCAC/AC/A (6). Of the four ORFs present in the Vir2 transcribed sequence, the region $5^{\prime}$ to the first ATG codon, TCAAA, fits most closely with this consensus sequence. The cryparin gene also contains a TCAAA sequence immediately upstream of the first ATG site, which has been shown to be the translation initiation site by protein $\mathrm{N}$-terminal analysis (57). It is likely that this consensus sequence is also the site of translation initiation for the Vir2 gene.

Although the sequence around the first ATG site appears to be a strong translation start signal on the basis of its sequence similarity to the fungal translational consensus sequence, the ORF following it encodes only 23 amino acids. Furthermore, the three in-frame ORFs following ORF 1 are also very short $(31,13$, and 11 amino acids, in length). No significant homologies were found in the EMBL data library for any of these ORFs. Interestingly, the C-terminal sequence encoded by ORF 1 contains a CAAX box (CVVM), where $C$ is cysteine, $A$ is an aliphatic amino acid, and $X$ is any amino acid. In many eukaryotic systems, including fungi, the CAAX motif predicts the site of posttranslational prenylation (48). Several fungal mating pheromones have been identified as prenylated lipopeptides (reviewed in references 48 and 50). Additionally, many ras oncogene proteins, ras-related small $G$ proteins, heterotrimeric $G$ proteins, and nuclear lamin proteins contain C-terminal CAAX motifs (50). The sequence encoded by the Vir2 ORF 1 also contains an asparagine at a site conserved in most known fungal propheromones (32). This asparagine in the ORF 1 product, amino acid 9 , likely predicts the $\mathbf{N}$ terminus of the processed pheromone, as was found in the Saccharomyces cerevisiae a-propheromone.

The codons used in the first ORF of Vir2, in comparison with those in the remaining ORFs, are in greatest agreement with the preferred codon usage of filamentous fungi (25). For example, serine is a common amino acid in the deduced sequence encoded by the first three ORFs of Vir2. Of 54 genes sequenced from 12 filamentous fungi, the codons used for serine are UCC (29.7\%), UCU (20.8\%), AGC (19.7\%), UCG (16.0\%), AGU (7.2\%), and UCA (6.8\%) (compiled from reference 25). In the first ORF of Vir2, all five serine residues are encoded by $\mathrm{UCC}$, the most common serine codon in fungal genes. In contrast, less frequently occurring serine codons are utilized in the second and third ORFs. Additionally, a putative $\mathrm{N}$-glycosylation site consisting of Asn-Ser-Ser (19) is present in ORF 1.

In summary, several structural features of ORF 1 suggest that it is translated: the presence of the first ATG codon, the common codon usage, a canonical translation initiation sequence, a potential $\mathrm{N}$-glycosylation site, and a CAAX box. However, it is possible that some other mechanism of gene regulation involving an RNA intermediate could account for the altered phenotype of the null mutant. For example, both the mouse $H 19$ gene (7) and the Drosophila heat shock response gene hsrw (23) are transcribed into RNA molecules that contain only very small ORFs; they are polyadenylated and spliced but not translated into detectable protein. Fini et al. (23) suggest that the cytoplasmic RNA w3 is translated but does not lead to detectable accumulation of the protein product. Instead, the act of translation itself may play a role in the regulation of cellular activities. At this 
point, it is unclear whether the final gene product of Vir2 is a protein or an RNA molecule.

The Vir2 null mutant partially mimics some of the hypovirulence-associated traits of dsRNA-containing strains. Sporulation and pycnidium production by the $\mathrm{pDH} 25$ control transformants were not significantly different from those of EP155/2. In contrast, spore and pycnidium numbers (assayed on two separate media) were reduced to as little as 0.1 and $22.1 \%$, respectively, of wild-type levels in $18 \mathrm{dm}$, in which the Vir2 gene was deleted by a site-specific integration event. Comparable reductions were observed at all time points examined, including 2, 3, and 4 weeks after plate inoculations (data not shown), suggesting that the effect was not due merely to a delay in development of $18 \mathrm{dm}$. Under the same growth conditions, the dsRNA-containing strain UEP1, in which Vir2 is down-regulated, failed to produce any detectable spores or pycnidia. Therefore, deletion of the Vir2 gene results in a phenotype that partially mimics one aspect of many dsRNA-containing strains of $C$. parasitica, i.e., a reduction in asexual sporulation. This reduction in the Vir2 null mutant appears to be caused primarily by a reduction in pycnidium number. However, it is unclear whether other processes that affect spore number, e.g., number of spores per pycnidium, are perturbed as well. Deletion of the Vir2 gene had no observable effect on pigmentation or virulence, two traits commonly altered by the presence of dsRNA.

The association of the reduction of asexual sporulation with disruption of the Vir2 gene was confirmed by restoration of the wild-type phenotype in a significant number of transformants (5 of 14) into which the Vir2 gene was introduced and in which its expression was detectable. Complementation of the Vir2 null mutation by pCV2 did not restore wild-type sporulation in all transformants, but it would be unrealistic to expect the randomly inserted gene with variable copy numbers to function perfectly in a developmental process. Southern blot analysis of the transformants showed that the linearized plasmid integrated into different locations in each of the transformants. The two transformants in which Vir2 mRNA was not detectable exhibited the low level of sporulation typical of $18 \mathrm{dm}$. In these transformants, Virl mRNA (see the last paragraph below) was expressed normally, indicating that lack of detection of Vir2 mRNA was not the result of RNA extraction or gel loading problems. These results confirm the role of Vir 2 in asexual sporulation and suggest that normal function of the gene is affected by its location within the genome. A variety of genetic abnormalities causing barren perithecia have been characterized by cytological examination of $N$. crassa mutants (39). From these studies, barrenness generally indicates an impairment in meiosis or ascus development. That inactivation of Vir2 affects both sexual and asexual sporulation is also of interest. A number of genes in $A$. nidulans have been implicated as playing roles in both asexual and sexual development (reviewed in reference 17). We are particularly intrigued by the presence of a putative prenylation site in ORF 1 of Vir2. The small size of ORF 1 and the presence of a CAAX motif at the $\mathrm{C}$ terminus of the predicted polypeptide are suggestive of the precursors of prenylated mating factors that have been described for several fungal species (see references cited in reference 32). That disruption of the Vir2 gene alters mating ability and outcome is supportive of this possibility. Studies are under way to further characterize the possible role of the Vir2 gene as a mating factor in $C$. parasitica.

The effect of deletion of Vir2 on both asexual sporulation and sexual mating is unique, particularly if $V i r 2$ is found to encode a pheromone. The biology of sporulation of $C$. parasitica is not inconsistent, however, with a single signal molecule being involved in both asexual and sexual sporulation. Where perithecia form, they are always found in the stroma below and after maturation of the pycnidia (42).

Reductions in pycnidium production and sporulation, and altered mating ability, are common traits associated with the hypovirulent phenotype of dsRNA-containing strains of $C$. parasitica. That deletion of the Vir2 gene resulted in only a partial loss of asexual sporulation and pycnidium production, in contrast to the near total suppression exhibited by the isogenic dsRNA-containing strain UEP1, suggests that the virus perturbs other genes that are redundant in function or are otherwise involved in these processes. To our knowledge, this is the first example of the partial reproduction of a virus-induced phenotype by deletion of a virus-perturbed host gene. The specific role of the Vir2 gene of $C$. parasitica in asexual and sexual sporulation is unknown at this time.

We have determined by Northern blots, S1 nuclease protection analyses, and DNA sequencing data that the Vir2 gene has homology with another $C$. parasitica gene, Vir1 (56). Deletion of the Vir2 gene does not affect the expression of Virl. Both genes are down-regulated by virus-encoded dsRNA, and Virl shows a time course of expression similar to that of Vir2. We have cloned the Virl gene and are in the process of characterizing it further by targeted gene disruption. We do not yet know the significance of the homology between Virl and Vir2, although it may suggest a similar function. Comparison of the structures of fungal genes regulated by virus-encoded dsRNA may offer clues as to the mechanism of dsRNA regulation of $C$. parasitica gene expression.

\section{ACKNOWLEDGMENTS}

We are grateful to D. Rigling for advice and assistance with the chestnut tree inoculations and to J. Vandenberg for assistance with statistical analyses of data. D. Ebbole and G. P. Podgorski provided helpful suggestions during the preparation of the manuscript.

This work was supported by grants from the National Science Foundation (MCB-9205818) and the USDA National Research Initiative (90-37290-5671).

\section{REFERENCES}

1. Anagnostakis, S. L. 1977. Vegetative incompatibility in Endothia parasitica. Exp. Mycol. 1:306-316.

2. Anagnostakis, S. L. 1979. Sexual reproduction of Endothia parasitica in the laboratory. Mycologia 71:213-215.

3. Anagnostakis, S. L. 1982. Biological control of chestnut blight. Science 215:466-471.

4. Anagnostakis, S. L. 1987. Chestnut blight: the classical problem of an introduced pathogen. Mycologia 79:23-37.

5. Ausubel, F. M., R. Brent, R. E. Kingston, D. D. Moore, J. G. Seidman, J. A. Smith, and K. Struhl. 1991. Current protocols in molecular biology. Greene Publishing and Wiley-Interscience, New York.

6. Ballance, D. J. 1991. Transformation systems for filamentous fungi and an overview of fungal gene structure, p. 1-29. In S. A. Leong and R. M. Berka (ed.), Molecular industrial mycology: systems and applications for filamentous fungi. Marcel Dekker, New York.

7. Brannan, C. I., E. C. Dees, R. S. Ingram, and S. M. Tilghman. 1990. The product of the H19 gene may function as an RNA. Mol. Cell. Biol. 10:28-36.

8. Carpenter, C. E., R. J. Mueller, P. Kazmierczak, L. Zhang, D. K. Villalon, and N. K. Van Alfen. 1992. Effect of a virus on accumulation of a tissue-specific cell-surface protein of the fungus Cryphonectria (Endothia) parasitica. Mol. Plant Microbe Interact. 5:55-61.

9. Choi, G. H., T. G. Larson, and D. L. Nuss. 1992. Molecular 
analysis of the laccase gene from the chestnut blight fungus and selective suppression of its expression in an isogenic hypovirulent strain. Mol. Plant Microbe Interact. 5:119-128.

10. Choi, G. H., and D. L. Nuss. 1992. A viral gene confers hypovirulence-associated traits to the chestnut blight fungus. EMBO J. 11:473-477.

11. Choi, G. H., and D. L. Nuss. 1992. Hypovirulence of chestnut blight fungus conferred by an infectious viral cDNA. Science 257:800-803.

12. Choi, G. H., D. M. Pawlyk, and D. L. Nuss. 1991. The autocatalytic protease p29 encoded by a hypovirulence-associated virus of the chestnut blight fungus resembles the potyvirus-encoded protease HC-Pro. Virology 183:747-752.

13. Choi, G. H., R. Shapira, and D. L. Nuss. 1991. Cotranslational autoproteolysis involved in gene expression from a doublestranded RNA genetic element associated with hypovirulence of the chestnut blight fungus. Proc. Natl. Acad. Sci. USA 88:11671171.

14. Churchill, A. C. L., L. M. Ciuffetti, D. R. Hansen, H. D. Van Etten, and N. K. Van Alfen. 1990. Transformation of the fungal pathogen Cryphonectria parasitica with a variety of heterologous plasmids. Curr. Genet. 17:25-31.

15. Cullen, D., S. A. Leong, L. J. Wilson, and D. J. Henner. 1987. Transformation of Aspergillus nidulans with the hygromycinresistance gene, $h p h$. Gene 57:21-26.

16. Day, P. R., J. A. Dodds, J. E. Elliston, R. A. Jaynes, and S. L. Anagnostakis. 1977. Double-stranded RNA in Endothia parasitica. Phytopathology 67:1393-1396.

17. Dean, R. A. 1992. Regulation of gene expression and signals in fungal development, p. 3-27. In D. P. S. Verma (ed.), Molecular signals in plant-microbe communications. CRC Press, Ann Arbor, Mich.

18. Del Sal, G., G. Manfioletti, and C. Schneider. 1988. A one-tube plasmid DNA mini-preparation suitable for sequencing. Nucleic Acids Res. 16:9878.

19. Devereux, J., P. Haeberli, and O. Smithies. 1984. A comprehensive set of sequence analysis programs for the VAX. Nucleic Acids Res. 12:387-395.

20. Dodds, J. A. 1980 . Association of type 1 viral-like dsRNA with club-shaped particles in hypovirulent strains of Endothia parasitica. Virology 107:1-12.

21. Elliston, J. E. 1985. Characteristics of dsRNA-free and dsRNAcontaining strains of Endothia parasitica in relation to hypovirulence. Phytopathology 75:151-158.

22. Fahima, T., P. Kazmierczak, D. R. Hansen, P. Pfeiffer, and N. K. Van Alfen. 1993. Membrane-associated replication of an unencapsidated double-strand RNA of the fungus, Cryphonectria parasitica. Virology 195:81-89.

23. Fini, M. E., W. G. Bendena, and M. L. Pardue. 1989. Unusual behavior of the cytoplasmic transcript of hsrw: an abundant, stress-inducible RNA that is translated but yields no detectable protein product. J. Cell Biol. 108:2045-2057.

24. Gritz, L., and J. Davies. 1983. Plasmid-encoded hygromycin B resistance: the sequence of hygromycin B phosphotransferase gene and its expression in Escherichia coli and Saccharomyces cerevisiae. Gene 25:179-188.

25. Gurr, S. J., S. E. Unkles, and J. R. Kinghorn. 1987. The structure and organization of nuclear genes of filamentous fungi, p. 93-139. In J. R. Kinghorn (ed.), Gene structure in eukaryotic microbes. IRL Press, Washington, D.C.

26. Hansen, D. R., N. K. Van Alfen, K. Gillies, and W. A. Powell. 1985. Naked dsRNA associated with hypovirulence of Endothia parasitica is packaged in fungal vesicles. J. Gen. Virol. 66:26052614.

27. Havir, E. A., and S. L. Anagnostakis. 1983. Oxalate production by virulent but not by hypovirulent strains of Endothia parasitica. Physiol. Plant Pathol. 23:369-376.

28. Hillman, B. I., R. Shapira, and D. L. Nuss. 1990. Hypovirulence-associated suppression of host functions in Cryphonectria parasitica can be partially relieved by high light intensity. Phytopathology 80:950-956.

29. Koonin, E. V., G. H. Coi, D. L. Nuss, R. Shapira, and J. C. Carrington. 1991. Evidence for common ancestry of a chestnut blight hypovirulence-associated double-stranded RNA and a group of positive-strand RNA plant viruses. Proc. Natl. Acad. Sci. USA 88:10647-10651.

30. Kozak, M. 1987. An analysis of 5 '-noncoding sequences from 669 vertebrate messenger RNAs. Nucleic Acids Res. 15:81258148.

31. L'Hostis, B. S. T. Hiremath, R. E. Rhoads, and S. A. Ghabrial. 1985. Lack of sequence homology between double-stranded RNA from European and American hypovirulent strains of Endothia parasitica. J. Gen. Virol. 66:351-355.

32. Moore, T. D. E., and J. C. Edman. 1993. The $\alpha$-mating type locus of Cryptococcus neoformans contains a peptide pheromone gene. Mol. Cell. Biol. 13:1962-1970.

33. Nuss, D. L., and Y. Koltin. 1990. Significance of dsRNA genetic elements in plant pathogenic fungi. Annu. Rev. Phytopathol. 28:37-58.

34. Paul, C. P., and D. W. Fulbright. 1988. Double-stranded RNA molecules from Michigan hypovirulent isolates of Endothia parasitica vary in size and sequence homology. Phytopathology 78:751-755.

35. Powell, W. A. (Syracuse University of New York). 1991. Personal communication.

36. Powell, W. A., and N. K. Van Alfen. 1987. Differential accumulation of poly $(\mathrm{A})^{+}$RNA between virulent and double-stranded RNA-induced hypovirulent strains of Cryphonectria (Endothia) parasitica. Mol. Cell. Biol. 7:3688-3693.

37. Powell, W. A., Jr., and N. K. Van Alfen. 1987. Two nonhomologous viruses of Cryphonectria (Endothia) parasitica reduce accumulation of specific virulence-associated polypeptides. J. Bacteriol. 169:5324-5326.

38. Puhalla, J. E., and S. L. Anagnostakis. 1971. Genetics and nutritional requirements of Endothia parasitica. Phytopathology 61:169-173.

39. Raju, N. B., and D. D. Perkins. 1978. Barren perithecia in Neurospora crassa. Can. J. Genet. Cytol. 20:41-59.

40. Rigling, D., U. Heiniger, and H. R. Hohl. 1989. Reduction of laccase activity in dsRNA-containing hypovirulent strains of Cryphonectria (Endothia) parasitica. Phytopathology 79:219 223.

41. Rigling, D., and N. K. Van Alfen. 1991. Regulation of laccase biosynthesis in the plant-pathogenic fungus Cryphonectria parasitica by double-stranded RNA. J. Bacteriol. 173:80008003

42. Roane, M. K., G. J. Griffin, and J. R. Elkins. 1986. Chestnut blight, other Endothia diseases, and the genus Endothia. APS Monograph Series. APS Press, St. Paul, Minn.

43. Roberts, A. N., V. Berlin, K. M. Hager, and C. Yanofsky. 1988. Molecular analysis of a Neurospora crassa gene expressed during conidiation. Mol. Cell. Biol. 8:2411-2418.

44. SAS Institute. 1985. SAS/STAT guide for personal computers. SAS Institute, Cary, N.C.

45. Shapira, R., G. H. Choi, B. I. Hillman, and D. L. Nuss. 1991. The contribution of defective RNAs to the complexity of viral-encoded double-stranded RNA populations present in hypovirulent strains of the chestnut blight fungus Cryphonectria parasitica. EMBO J. 10:741-746.

46. Shapira, R., G. H. Choi, and D. L. Nuss. 1991. Virus-like genetic organization and expression strategy for a double-stranded RNA genetic element associated with biological control of chestnut blight. EMBO J. 10:731-739.

47. Shapira, R., and D. L. Nuss. 1991. Gene expression by a hypovirulence-associated virus of the chestnut blight fungus involves two papain-like protease activities. J. Biol. Chem. 266:19419-19425.

48. Sinensky, M., and R. J. Lutz. 1992. The prenylation of proteins. Bioessays 14:25-31.

49. Smale, S. T., and D. Baltimore. 1989. The "initiator" as a transcription control element. Cell 57:103-113.

50. Stimmel, J. B., R. J. Deschenes, C. Volker, J. Stock, and S. Clarke. 1990. Evidence for an S-farnesylcysteine methyl ester at the carboxyl terminus of the Saccharomyces cerevisiae Ras 2 protein. Biochemistry 29:9651-9659.

51. Tartaglia, J., C. P. Paul, D. W. Fulbright, and D. L. Nuss. 1986. 
Structural properties of double-stranded RNAs associated with biological control of chestnut blight fungus. Proc. Natl. Acad. Sci. USA 83:9109-9113.

52. Van Alfen, N. K. 1986. Hypovirulence of Endothia (Cryphonectria) parasitica and Rhizoctonia solani, p. 143-162. In K. W. Buck (ed.), Fungal virology. CRC Press, Boca Raton, Fla.

53. Varley, D. A., G. K. Podila, and S. T. Hiremath. 1992. Cutinase in Cryphonectria parasitica, the chestnut blight fungus: suppression of cutinase gene expression in isogenic hypovirulent strains containing double-stranded RNAs. Mol. Cell. Biol. 12: $4539-4544$.
54. Vollmer, S. J., and C. Yanofsky. 1986. Efficient cloning of genes of Neurospora crassa. Proc. Natl. Acad. Sci. USA 83:48694873.

55. Yelton, M. M., J. E. Hamer, and W. E. Timberlake. 1984. Transformation of Aspergillus nidulans by using a trpC plasmid. Proc. Natl. Acad. Sci. USA 81:1470-1474.

56. Zhang, L., and N. K. Van Alfen. Unpublished data.

57. Zhang, L., D. Villalon, Y. Sun, P. Kazmierczak, and N. K. Van Alfen. Virus-associated down-regulation of the gene encoding cryparin, an abundant cell-surface protein from the chestnut blight fungus, Cryphonectria parasitica. Gene, in press. 